\title{
BMJ Open Effectiveness and safety of modified 'Huoxue Shugan' formulas on coronary heart disease combined with depression: protocol for a systematic review
}

\author{
Mingtai Chen, ${ }^{1}$ Ling Men, ${ }^{2}$ Lijun Ou, ${ }^{1}$ Tao Li, ${ }^{1}$ Meihuan Li, ${ }^{1}$ Xiaoling Zhong, ${ }^{3}$ \\ Jian Zhang, ${ }^{4}$ Zhong Zhang ${ }^{1}$
}

To cite: Chen M, Men L, Ou L, et al. Effectiveness and safety of modified 'Huoxue Shugan' formulas on coronary heart disease combined with depression: protocol for a systematic review. BMJ Open 2018;8:e022868. doi:10.1136/ bmjopen-2018-022868

- Prepublication history for this paper is available online. To view these files, please visit the journal online (http://dx.doi. org/10.1136/bmjopen-2018022868).

MC and LM contributed equally.

Received 22 March 2018 Revised 4 October 2018 Accepted 5 October 2018

Q Check for updates

(c) Author(s) (or their employer(s)) 2018. Re-use permitted under CC BY-NC. No commercial re-use. See rights and permissions. Published by BMJ.

For numbered affiliations see end of article.

Correspondence to Professor Xiaoling Zhong; szszyyzx|@163.com, Professor Jian Zhang;

fuwaixinshuai@163.com and

Professor Zhong Zhang;

179080693@qq.com

\section{ABSTRACT}

Objective To assess the clinical effectiveness and safety of modified 'Huoxue Shugan' (HXSG) formulas used as Chinese herbal medicine in treating patients with coronary heart disease (CHD) and depression.

Methods A systematic literature search of articles up to March 2018 will be performed in the following electronic databases: PubMed, Embase, the Cochrane Library, China National Knowledge Infrastructure, Chinese Scientific Journals Database, Chinese Biomedical Database, Chinese Biomedical Literature Service System and Wanfang Database. Inclusion criteria are as follows: randomised controlled trials of modified HXSG formulas in patients with $\mathrm{CHD}$ and depression. The primary outcome measures will be CHD-related clinical evaluation (frequency of acute angina, severity of angina pectoris, ECG changes, dose of nitroglycerin) and the scores or amount of reduction in scales measuring depression (ie, the Hamilton Depression Scale or other widely used depression scales). The safety outcome measures will be adverse events, liver and kidney function. RevMan V.5.3 software will be used for data synthesis, sensitivity analyses, subgroup analyses and risk of bias assessment. A funnel plot will be developed to evaluate reporting bias. Stata V.12.0 will be used for meta-regression and Egger tests. We will use the Grading of Recommendations Assessment, Development and Evaluation system to assess the quality of evidence. Ethics and dissemination This systematic review does not require ethics approval and will be submitted to a peer-reviewed journal.

PROSPERO registration number CRD42018089641.

\section{INTRODUCTION}

Coronary heart disease (CHD) has remained one of the most serious diseases; it has high mortality and morbidity and is the leading cause of death worldwide. ${ }^{1}$ Depression has been another concerning mental health problem, leading to serious disability, and has largely contributed to the overall global burden of disease. ${ }^{2}$ It has been widely reported that depression can aggravate CHD-related angina symptoms and lead to an adverse CHD prognosis. Conversely, CHD
Strengths and limitations of this study

- This study is an objective and normative systematic review evaluating the effectiveness and safety of modified 'Huoxue Shugan' formulas used as Chinese herbal medicine in the treatment of patients with coronary heart disease and depression.

- Study screening, data extraction and assessment of the risk of bias will be independently conducted by two researchers.

- Disagreements will be resolved by discussion or arbitrated by a third author if needed.

- There may be clinical heterogeneity because of variations in treatment frequency or duration, or other factors. Therefore, subgroup analyses or meta-regression analyses will be carried out to explore any source of heterogeneity.

- The Grading of Recommendations Assessment, Development and Evaluation system will be used to further evaluate study findings.

- Because this is a retrospective meta-analysis, the search has the inevitable potential of excluding unpublished trials which will introduce some bias.

has also been shown to aggravate depressive symptoms. ${ }^{3}$ In the population of patients with CHD, depression has not only been linked to a twofold increase in the risk of death and a higher risk for major adverse cardiac events but has also been shown to diminish longterm quality of life. ${ }^{4-6}$ According to one study, approximately $40 \%$ of patients with CHD have comorbid depression and represent the major group experiencing worse physical health outcomes associated with CHD when compared with similar patients with CHD but without depression. ${ }^{7}$

Because of the association between CHD and depression, CHD basic treatment and antidepressant therapy have always been administered in parallel in the clinical arena. However, the side effects of antidepressants 
has limited their use overall in the long-term treatment of patients with depression. ${ }^{8}$

Chinese herbal medicine (CHM) has been used clinically as therapy treatment for thousands of years. Currently, CHM is commonly and widely used as alternative therapy for the management of CHD and depression in China. There is increasing evidence that therapy with CHM has emerged as a potentially promising therapeutic measure for patients with CHD and depression. 9-11 Previous studies have demonstrated that 'Huoxue' formulas play a significant role in relieving angina pectoris and improving ECG results in patients with CHD. ${ }^{12-14}$ Moreover, 'Shugan' formulas, which have been used most commonly in depressed patients, have been shown to significantly improve depressive symptoms. ${ }^{15} 16$ Among a variety of CHM therapies, modified 'Huoxue Shugan' (HXSG) formulas have been employed in clinical medicine most commonly in patients with CHD and depression. $^{917}$

Despite numerous previous clinical studies and reviews evaluating CHM therapies in the treatment of patients with $\mathrm{CHD}$ and depression, systematic reviews to assess the effectiveness and safety of different HXSG formulas in this patient population are rare. We identified only one meta-analysis evaluating the effectiveness of CHM therapies in patients with CHD and depression, though the study had certain limitations. ${ }^{8}$ There were 13 randomised clinical trials (RCTs) included in the meta-analysis by Wang et al. ${ }^{8}$ However, the CHM therapies studied were both dissimilar and complex; there was heterogeneity among the interventions, and the outcomes of the CHD-related clinical evaluations were relatively incomplete.

In view of the shortcomings of previous studies and the insufficient evidence regarding the widespread use of HXSG formulas, this systematic review aimed to summarise the effectiveness and safety of modified HXSG formulas, one of the important CHM therapies, in treating patients with $\mathrm{CHD}$ and depression.

\section{METHODS AND ANALYSIS Registration}

The study protocol has been registered in the international prospective register of systematic review (PROSPERO). The procedure of this protocol will be conducted according to the Preferred Reporting Item for Systematic Reviews and Meta-Analyses Protocols (PRISMA-P) guidelines. ${ }^{18}$

\section{Eligibility criteria}

Type of study

\section{Inclusion}

We will include all the RCTs that investigated the effectiveness and safety of modified HXSG formulas combined with pharmacotherapy for the treatment of patients with CHD and depression.

\section{Exclusion}

The studies will be excluded if it is not an RCT (namely, observational cohort and case-control studies, case reports, experimental studies and reviews).

\section{Participants}

Inclusion

The study will include patients diagnosed simultaneously with both CHD and depression regardless of age, sex, ethnicity, education or economic status and whether or not they were outpatients or inpatients. The diagnostic criteria for $\mathrm{CHD}$ and depression will be as follows ${ }^{19-21}$ :

1. 1.The diagnostic criteria of CHD should be confirmed according to one of the past or current definitions: Report of the Joint International Society and Federation of Cardiology/WHO task force on standardisation of clinical nomenclature of ischaemic heart disease, or the American College of Cardiology/American Heart Association guideline update for the management of patients with chronic stable angina or Chinese Association of Cardiology or unstable angina pectoris diagnosis and treatment recommendations. ${ }^{22-24}$

2. 2.Depression must be defined as a depressive disorder or clinical depression diagnosed according to the Diagnostic and Statistical Manual of Mental Disorders, the International Classification of Diseases by a standardised interview (eg, Structured Clinical Interview, Composite International Diagnostic Interview) or the Chinese Classification of Mental Disorders.

\section{Exclusion}

Patients with either CHD or depression only will be excluded. Patients with severe respiratory disease, acute infectious disease, severe heart disease, severe liver disease or tumours will be excluded.

\section{Interventions}

\section{Inclusion}

Eligible interventions will be those involving a combination of modified HXSG formulas and conventional pharmacotherapy. The same conventional pharmacotherapy must be used in the control group.

\section{Exclusion}

Trials that include other cointerventions such as another herbal formula, acupuncture, cupping, moxibustion, massage, yoga, qigong, Tai Chi or aromatherapy will be excluded.

\section{Outcome}

\section{Inclusion}

The primary outcome measures will include the following: CHD-related clinical evaluation (frequency of acute angina, severity of angina pectoris, ECG changes, dose of nitroglycerin), the scores or reduction in scales measuring depression (ie, the Hamilton Depression Scale or other widely used depression scale). ${ }^{25}$ The secondary outcome measures will include the following: total cholesterol, triglyceride, low-density lipoprotein 
cholesterol and high-density lipoprotein cholesterol levels and the Traditional Chinese Medicine syndrome scale. The safety outcomes will include the following: adverse events (such as digestive symptoms, headache, dizziness, skin rash, etc), liver or kidney toxicity measured by serum markers.

\section{Exclusion}

The outcome measures not requested in this study will be excluded.

\section{Search strategy}

The following electronic bibliographic databases will be searched from inception to March 2018: PubMed, Embase, the Cochrane Library, China National Knowledge Infrastructure, Chinese Scientific Journals Database, Chinese Biomedical Database, Chinese Biomedical Literature Service System and Wanfang Database. A manual search of key journals and of the reference lists of reviews captured by the initial searches will also be performed. There will be no limits on the language of publication. Only clinical trials will be included and searched. The following sources will also be searched to identify clinical trials that are in progress or completed: Clinical Trials. gov and WHO clinical trials registry. Any additional relevant studies will also be retrieved from the reference lists of systematic reviews and included studies. If possible, we will map search terms to controlled vocabulary. In addition, the search strategy for selecting the fields of title, abstract or keyword will differ depending on the characteristics of the databases. Search terms will be grouped into three blocks (see table 1 ).

\section{Study selection and data extraction}

Literature-retrieved citations will be managed by EndNote X7 software. Two authors (MC and ML) will independently screen the titles and abstracts of all the studies retrieved in the above electronic databases to identify potentially eligible studies. Articles that are duplicated or have not met the eligibility criteria, interventions and outcomes in this study will be excluded. After filtering the final eligible articles, the data from the included articles will be extracted independently by two authors (MC and ML). Disagreements will be resolved by discussion or arbitrated by a third author if needed (LM or ZZ). The following categories of data will be extracted: first author, publication year, diagnose information, age, sex, trial characteristics, interventions and controls, participants, study methodology, outcomes and adverse events (see figure 1).

\section{Risk of bias assessment}

The methodological quality of the eligible studies will be evaluated according to the Cochrane Collaboration's tool for assessing risk of bias. ${ }^{26}$ The assessment details include: sequence generation, allocation concealment, blinding of participants and personnel, blinding of outcome assessors, incomplete outcome data, selective reporting and other sources of bias. Each domain will be assessed as 'low risk', 'high risk' or 'unclear risk' according to the description details of eligible studies.

\section{Data synthesis and statistical analysis}

Statistical analyses will be conducted with RevMan V.5.3 software provided by Cochrane Collaboration. The

Table 1 Search items

\begin{tabular}{|c|c|}
\hline Search block & Search items \\
\hline Participants & $\begin{array}{l}\text { (Coronary Disease OR Myocardial Ischemia OR Coronary Diseases OR Disease, Coronary OR Diseases, } \\
\text { Coronary OR Coronary Heart Disease OR Coronary Heart Diseases OR Disease, Coronary Heart OR } \\
\text { Diseases, Coronary Heart OR Heart Disease, Coronary OR Heart Diseases, Coronary OR CHD OR } \\
\text { Myocardial Infarction OR Cardiovascular Stroke OR Heart Attack OR Myocardial Infarct OR Acute Coronary } \\
\text { Syndrome OR Angina Pectoris OR Angina, Stable OR Angina, Unstable OR Angina Pectoris, Variant OR } \\
\text { Microvascular Angina) } \\
\text { AND } \\
\text { (Depressions OR Depressive Symptoms OR Depressive Symptom OR Symptom, Depressive OR } \\
\text { Symptoms, Depressive OR Emotional Depression OR Depression, Emotional OR Depressions, Emotional } \\
\text { OR Emotional Depressions OR Depressive Disorders, Major OR Depressive Disorders OR Depressive } \\
\text { Syndrome OR) }\end{array}$ \\
\hline Intervention & $\begin{array}{l}\text { Shugan OR Huoxue OR Huoxue Shugan decoction OR Huoxue Shugan formula OR Huoxue Shugan } \\
\text { tang OR Drugs, Chinese Herbal OR Chinese Drugs, Plant OR Chinese Herbal Drugs OR Herbal Drugs, } \\
\text { Chinese OR Plant Extracts, Chinese OR Chinese Plant Extracts OR Extracts, Chinese Plant OR Medicine, } \\
\text { Chinese Traditional OR Traditional Chinese Medicine OR Chung I Hsueh OR Hsueh, Chung I OR Traditional } \\
\text { Medicine, Chinese OR Zhong Yi Xue OR Chinese Traditional Medicine OR Chinese Medicine, Traditional } \\
\text { OR Traditional Tongue Diagnosis OR Tongue Diagnoses, Traditional OR Tongue Diagnosis, Traditional OR } \\
\text { Traditional Tongue Diagnoses OR Traditional Tongue Assessment OR Tongue Assessment, Traditional OR } \\
\text { Traditional Tongue Assessments OR }\end{array}$ \\
\hline Study design & $\begin{array}{l}\text { Randomized controlled trial OR controlled clinical trial OR randomized OR placebo OR drug therapy OR } \\
\text { randomly OR trial OR groups }\end{array}$ \\
\hline
\end{tabular}

CHD, coronary heart disease. 


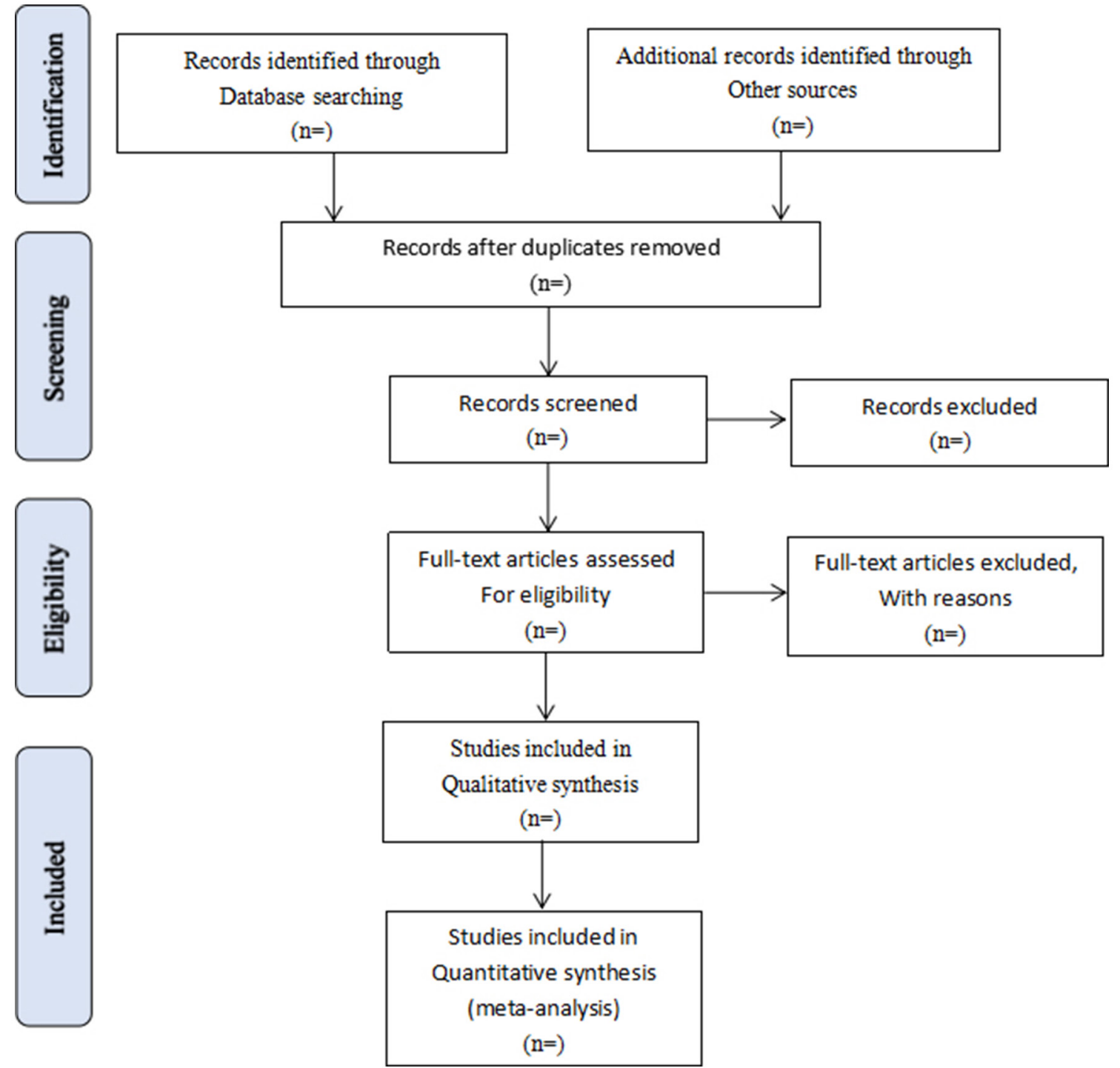

Figure 1 Flow diagram of study selection process. PubMed, Embase, the Cochrane Library, China National Knowledge Infrastructure, Chinese Scientific Journals Database, Chinese Biomedical Database, Chinese Biomedical Literature Service System and Wanfang Database.

overall effect sizes will be determined as the mean difference for continuous outcomes, the OR for dichotomous outcomes with their $95 \%$ credible intervals. The $Q$ and $\mathrm{I}^{2}$ test statistics will be calculated to determine the amount of heterogeneity. For the $\mathrm{Q}$ statistic, $\mathrm{p}<0.05$ will be considered to indicate significant differences. For the $\mathrm{I}^{2}$ statistic, $\mathrm{I}^{2}<25 \%$ indicates no significant heterogeneity, $\mathrm{I}^{2}=25 \%-$ $50 \%$ is considered moderate heterogeneity and $\mathrm{I}^{2}>50 \%$ indicates strong heterogeneity. We will use fixed effects models if there is no heterogeneity among studies, and random effects models if there is heterogeneity.

\section{Sensitivity analysis, subgroup analysis and meta-regression}

If the heterogeneity or inconsistency among the studies is detected, a sensitivity analysis or subgroup analysis or meta-regression (conducted by Stata V.12.0) analysis will be performed. Subgroup analysis will be conducted to explore potential sources of heterogeneity according to the characteristics of studies, including sample size, types of CHD, severity of depression, dose of HXSG formulas, treatment duration and other relevant parameters. If data extraction is insufficient, we will create a qualitative synthesis.

\section{Publication bias}

A funnel plot will be developed to evaluate reporting bias of the included studies. We will use Egger tests (conducted using Stata V.12.0) to assess funnel plot symmetry and will interpret values of $\mathrm{p}<0.1$ as statistically significant.

\section{Quality of evidence}

We will also assess the quality of evidence for the main outcomes with the Grading of Recommendations Assessment, Development and Evaluation approach. Five items will be investigated, including limitations in study design, inconsistency, inaccuracies, indirectness and publication bias. 


\section{Patient and public involvement}

The patients and/or public will not be involved because this study uses secondary sources for analysis.

\section{DISCUSSION}

We plan to conduct this meta-analysis to evaluate the effectiveness and safety of HXSG formulas for patients with CHD and depression. However, there may be some limitations because this is a retrospective meta-analysis. First, during the search, there is the inevitable potential that unpublished studies will not be identified which will introduce some bias. Moreover, some grey literature may be difficult to retrieve, possibly leading to a selection bias in the literature. In addition, some secondary outcome measures may not be completely reported. Nevertheless, we expect that the results of this study will be able to propose clinical recommendations for patients with CHD and depression in clinical practices that employ CHM and provide more reliable evidence supporting use of the latter.

\section{Ethics and dissemination}

The results of the meta-analysis will be reported according to the PRISMA extension statement and disseminated in a peer-reviewed journal.

\section{Author affiliations}

${ }^{1}$ Department of Cardiovascular Disease, Shenzhen Traditional Chinese Medicine Hospital, Guangzhou University of Chinese Medicine, Shenzhen, China ${ }^{2}$ Nephrology Department, Shenzhen Traditional Chinese Medicine Hospital, Guangzhou University of Chinese Medicine, Shenzhen, China ${ }^{3}$ Reproductive Health Department, Shenzhen Traditional Chinese Medicine Hospital, Guangzhou University of Chinese Medicine, Shenzhen, China

${ }^{4}$ Heart Failure Center, Fuwai Hospital Chinese Academy of Medical Sciences, Beijing, China

Contributors ZZ and JZ conceived the study and drafted the protocol. LM, L0 and $T L$ revised it. MC and ML developed the search strategies, will conduct data collection and analyse the data independently. All authors will approve the final manuscript.

Funding This study is supported by National Natural Science Foundation of China, NFSC (No.81573922); Sanming Project of Medicine in Shenzhen - Chinese Academy of Medical Sciences Fuwai Hospital Professor Zhang Jian Cardiovascular Disease Team (No.SZSM201612033)

Competing interests None declared.

Patient consent Not required.

Ethics approval This review does not require ethical approval because there are no concerns about patient privacy.

Provenance and peer review Not commissioned; externally peer reviewed.

Open access This is an open access article distributed in accordance with the Creative Commons Attribution Non Commercial (CC BY-NC 4.0) license, which permits others to distribute, remix, adapt, build upon this work non-commercially, and license their derivative works on different terms, provided the original work is properly cited, appropriate credit is given, any changes made indicated, and the use is non-commercial. See: http://creativecommons.org/licenses/by-nc/4.0/.

\section{REFERENCES}

1. World Health Organization. The top 10 causes of death. Fact sheet No.310. 2017. http://www.who.int/mediacentre/factsheets/fs310/en/ (Updated Jan 2017).

2. World Health Organization. Depression. Fact sheet No.369. 2017. http://www.who.int/mediacentre/factsheets/fs369/en/
3. Wu Q, Kling JM. Depression and the Risk of Myocardial Infarction and Coronary Death: A Meta-Analysis of Prospective Cohort Studies. Medicine 2016;95:e2815.

4. Barth J, Schumacher M, Herrmann-Lingen C. Depression as a risk factor for mortality in patients with coronary heart disease: a metaanalysis. Psychosom Med 2004;66:802-13.

5. Lichtman JH, Froelicher ES, Blumenthal JA, et al. Depression as a risk factor for poor prognosis among patients with acute coronary syndrome: systematic review and recommendations: a scientific statement from the American Heart Association. Circulation 2014;129:1350-69.

6. Samartzis L, Dimopoulos S, Tziongourou M, et al. Effect of psychosocial interventions on quality of life in patients with chronic heart failure: a meta-analysis of randomized controlled trials. $J$ Card Fail 2013;19:125-34.

7. Dickens C. Depression in people with coronary heart disease: prognostic significance and mechanisms. Curr Cardiol Rep 2015;17:83.

8. Wang AL, Chen Z, Luo J, et al. Systematic review on randomized controlled trials of coronary heart disease complicated with depression treated with Chinese herbal medicines. Chin J Integr Med 2016;22:56-66.

9. Ding HY, Wang BH, Xu Q. Current status of Chinese medicine research on coronary heart disease and depression. The $\mathrm{J}$ of Med Theory and Practice 2017;30:332-4.

10. Yeung WF, Chung KF, Ng KY, et al. A systematic review on the efficacy, safety and types of Chinese herbal medicine for depression. $J$ Psychiatr Res 2014;57:165-75.

11. Tian PP, Li J, Gao J, et al. Efficacy and safety of the shexiang baoxin pill for the treatment of coronary artery disease not amenable to revascularisation: study protocol for a randomised, placebocontrolled, double-blinded trial. BMJ Open 2018;8:e018052.

12. Jia Y, Leung SW. How efficacious is danshen (Salvia miltiorrhiza) dripping pill in treating angina pectoris? Evidence assessment for meta-analysis of randomized controlled trials. J Altern Complement Med 2017;23:676-84.

13. Huang J, Tang X, Ye F, et al. Clinical Therapeutic effects of aspirin in combination with fufang danshen diwan, a traditional chinese medicine formula, on coronary heart disease: a systematic review and meta-analysis. Cell Physiol Biochem 2016;39:1955-63.

14. Mao C, Fu XH, Yuan JQ, et al. Tong-xin-luo capsule for patients with coronary heart disease after percutaneous coronary intervention. Cochrane Database Syst Rev 2015;5:CD010237.

15. Liu W, Qin J. Clinical efficacy and safety of the Shugan Jieyu capsule in patients with acute myocardial infarction and depression. Int $J$ Psychiatry Med 2016;51:534-43.

16. Zhang X, Kang D, Zhang L, et al. Shuganjieyu capsule for major depressive disorder (MDD) in adults: a systematic review. Aging Ment Health 2014;18:941-53.

17. Wang Y, Fan R, Huang X. Meta-analysis of the clinical effectiveness of traditional Chinese medicine formula Chaihu-Shugan-San in depression. J Ethnopharmacol 2012;141:571-7.

18. Moher $D$, Shamseer $L$, Clarke $M$, et al. Preferred reporting items for systematic review and meta-analysis protocols (PRISMA-P) 2015 statement. Syst Rev 2015;4:1.

19. Wilkins LW. Nomenclature and criteria for diagnosis of ischemic heart disease. Report of the Joint International Society and Federation of Cardiology/World Health Organization task force on standardization of clinical nomenclature. Circulation 1979;59:607-9.

20. Gibbons RJ, Abrams J, Chatterjee K, et al. ACC/AHA 2002 guideline update for the management of patients with chronic stable angina-summary article: a report of the american college of cardiology/American heart association task force on practice guidelines (Committee on the Management of Patients W. Circulation 1999;33:2032.

21. Chinese Journal of Cardiology Editorial Committee. Diagnosis and treatment of unstable angina pectoris. Chinese Journal of Cardiology 2000;28.

22. Association A P. The diagnostic and statistical manual of mental disorders, DSM-IV. Encyclopedia of the Neurological Sciences 2000;25:4-8.

23. World Health Organization. International statistical classification of diseases and related health problems (10th revision). http://www. who.int/classifications/icd/en/

24. Chinese Society of Psychiatry. Chinese classification of mental disorders. Jinan: Shandong Science and Technology Press 2001:87-9.

25. Hamilton MJ. Neurology neurosurgery and psychiatry. 1960;23:56-62.

26. Higgins JPT, Green S. Cochrane handbook for systematic reviews of interventions Version5.1.0, 2011. http://www.handbook.cochrane.org 